\title{
Mask-less lithography for fabrication of optical waveguides
}

\author{
M. Dubov ${ }^{*}$, S. R. Natarajan, J.A.R. Williams and I. Bennion \\ Aston University, Birmingham B4 7ET, United Kingdom
}

\begin{abstract}
A flexible method for fabricating shallow optical waveguides by using femtosecond laser writing of patterns on a metal coated glass substrate followed by ion-exchange is described. This overcomes the drawbacks of low index contrast and high induced stress in waveguides directly written using low-repetition rate ultrafast laser systems. When compared to conventional lithography, the technique is simpler and has advantages in terms of flexibility in the types of structures which can be fabricated.
\end{abstract}

Keywords: ion-exchange, mask-less, femtosecond lithography, planar lightwave circuit

\section{INTRODUCTION}

Optical integration is an attractive method for not only reducing the cost and size of communication and sensing systems but also for efficient and optimal performance [1, 2]. Planar Lightwave Circuit (PLC) technology which forms the backbone of this integration, if applied with proper design, would result in replacing almost all bulk-optic hermetical packages with improved tolerance and saving of costly alignment hours [3]. Furthermore, it greatly reduces production stages while utilising wafer level semiconductor production technologies.

In this work we have implemented a combinational (or hybrid) technology for the production of shallow, 2D-arbitraryshaped, high refractive- index-contrast, and low propagation loss waveguides. The technique is flexible, low cost and suitable both for prototyping and mass production.

There are a number of PLC technologies available today, such as ion-exchange, polymer, sol-gel, direct-laser writing (with excimer and/or ultrafast lasers), diffusion/lithography/poling in Lithium Niobate, silica-on-silicon, silicon-oninsulator, silicon oxy-nitride-on-silicon, and epitaxy (GaAs, InP). The general stages of these technologies involve the design of a mask and delineation (image transfer) using standard photolithographic techniques in a clean room, the sole exception being a direct-laser writing. Each of these has some merits and demerits over the others [1]. Among the list, the most competitive technology, to our knowledge, is ion-exchange [4]. Polymer waveguides provide potentially low cost fabrication methods [5] while the sol-gel method, in addition to low cost, provides flexibility [6]. Direct-laser write (and two-photon polymerisation) is a recent technology and offers to reduce elaborate photolithography activity [7]. The rest of the techniques would require high processing temperature and/or complex deposition methodology.

In addition to being low-cost, the ion-exchange method produces low-loss $(<0.1 \mathrm{~dB} / \mathrm{cm})$ optical waveguides which have low sensitivity to polarisation, controlled mode shape, and, in principle, controlled depth of position by virtue of electric field assisted indiffusion. As compared with polymers they are more stable and can be easily coupled with conventional single mode fibres. The only problem remains is the required lithography. We use femtosecond direct laser writing to substitute conventional lithography. This can provide sub-micrometer resolution similar to good vacuum lithography and is also flexible and can be used to make masks in real time. Furthermore, this technology can be used to create periodic structures with a sub-micrometer period and offers the possibility of inscribing both waveguide and a sub-micrometer periodic perturbation simultaneously.

To the best of our knowledge, there has been little work reported using a combination of these techniques with ultrashort laser. Salguerio, et al [8], and Koch, et al [9, 10] have employed conventional photolithography to create masks but with the aid of direct laser writing on photoresist coating on metal layer. This method still requires consumables, such as photoresist and would result in high costs and long processing time for low volume production. We could refer to the problems of the photoresist exposure with NIR femtosecond lasers and photoresist heating by the pulse train, described

*m.dubov@aston.ac.uk; phone +44 121 2043495; fax +44 121 2043682; aston.ac.uk 
in [9]. Similar recent results on post-inscription of Bragg reflectors on top of ion-exchanged waveguides, demonstrated by Grobnic, et al [11] where a $1.6 \mu \mathrm{m}$ period structure was inscribed by mask method require a time-consuming additional sample alignment. In our work, we have combined the advantages of ion-exchange method with direct laser writing on (or ablation of) a thin metal Al layer, deposited on a glass surface.

A focused laser beam of NIR light at the wavelength of $800 \mathrm{~nm}$ is used to ablate a $100 \mathrm{~nm}$ thick metal layer of $\mathrm{Al}$ along a pre-defined path leaving the glass beneath exposed. Ideally, the surface of the glass should not be affected by laser ablation at all.

The main advantage of our method over previously reported techniques is that there is no need to specially treat the substrate with a photoresist or other photosensitive layer. This significantly reduces the processing steps and the need for additional consumables, and thus time for device prototyping. Glass slides pre-coated with a metal layer are commercially available. The whole manufacturing cycle (process) consists of the following stages: i) sample cleaning, ii) alignment and femtosecond laser ablation of the desired pattern, iii) post-laser-inscription clearing to remove possible debris, but often this could be omitted, if ablation protocol has already been optimised, iv) ion-exchange in a bath of melted salt(s) of Ag, v) removal of metal coating and final cleaning, vi) edge-polishing or cleaving for butt coupling and testing. The time consuming and delicate "polishing for coupling" stage could be removed if the PLC is designed for other than butt-coupling interconnection or is to be integrated into the photonics device. Post-treatments such as field assisted shaping and positioning (deepening) of the light guiding core at the designed depth or the introduction of additional surface corrugations by a laser on another run are, in principle, possible. The issues relating to direct femtosecond laser ablation of metal films, such as burr formation around the hole, microbumps and nanojets for lower laser pulse energies [9], highly nonlinear or even bi-stable absorption regimes with the dependence of the ablation depth on the protocol of laser illumination [12], and, of course, film (and its adhesion) non-uniformity, have been addressed in this work.

A number of approaches (such as using back illumination) were tested with the best results obtained using multiple scans and front illumination. These scans were made using a motorised stage capable of nanometer precision movements and laser pulse energies successively decreasing with each scan. The exposure was synchronised with stage motion to obtain sharp sub-micron scale features comparable to what is achievable using standard lithography. In our initial experiments we used an infrared camera to the transmitted laser light below the sample to manually monitor the ablation process. However, the implementation of a fully automatic optical feedback system would significantly improve the quality, robustness and precision of the ablation stage.

Ion-exchange was then performed by immersing the delineated patterned substrate in chemical bath under controlled conditions, to form waveguides. The metal layer was then removed and the samples were cut and edge-polished to couple light. Alternative design of input/output ports for optimal coupling with fibres is under consideration, which might significantly reduce this time consuming stage.

The ion exchanged structure has formed near the substrate surface and therefore can be used for a variety of sensing applications. If necessary, additional treatment such as coating could also be used. Special packaging of these devices can tailor their environmental sensitivity.

\section{EXPERIMENT}

Standard soda-lime glass microscopic slides [13] were used for the fabrication of ion-exchange waveguides. The glass was systematically cleaned in an ultrasonic bath to remove dirt, grease, organic and inorganic impurities. The cleaned samples were then coated with an Al coating in a clean room by thermal evaporation in a vacuum chamber under controlled conditions. The thickness of the coating was varied between 50 and $1000 \mathrm{~nm}$. Femtosecond Ti:Sapphire laser was used to ablate aluminium regions in the form of desired patterns suitable for producing windows (strips on the metal layer) through which ion-exchange was later carried out.

The laser setup consisted of a Kerr-lens mode-locked Ti:Sapphire oscillator (Tsunami), and regenerative chirped-pulse amplification system (Spitfire, both Spectra Physics). The $1 \mathrm{kHz}$ repetition rate femtosecond system delivers up to 0.75 $\mathrm{mJ}$ output pulses of less than $150 \mathrm{fs}$ pulsewidth at $800 \mathrm{~nm}$. The laser pulses were first coarsely attenuated with a Brewster angle thin-film polariser followed the zero-order half-waveplate (Fig. 1). The fine attenuation was finally achieved by computer controlled second half-waveplate and a Glan laser prism. The motorised stage (ABL-10050, Aerotech) held the sample and was capable of precise movements along a 2-dimensional plane. Both short and long term 
positioning errors (accuracy) of the stage were below $50 \mathrm{~nm}$. The femtosecond pulses were focused using a long working distance (x36) microscope objective with a numerical aperture of 0.5 . The diameter of the focused beam was estimated to be about $2 \mathrm{um}$. While this may be a limiting factor for a sub-micrometer periodical patterns ablation on a metal film, it was however possible to ablate in a single shot a hole as small as $100 \mathrm{~nm}$ in diameter using this microscope objective.

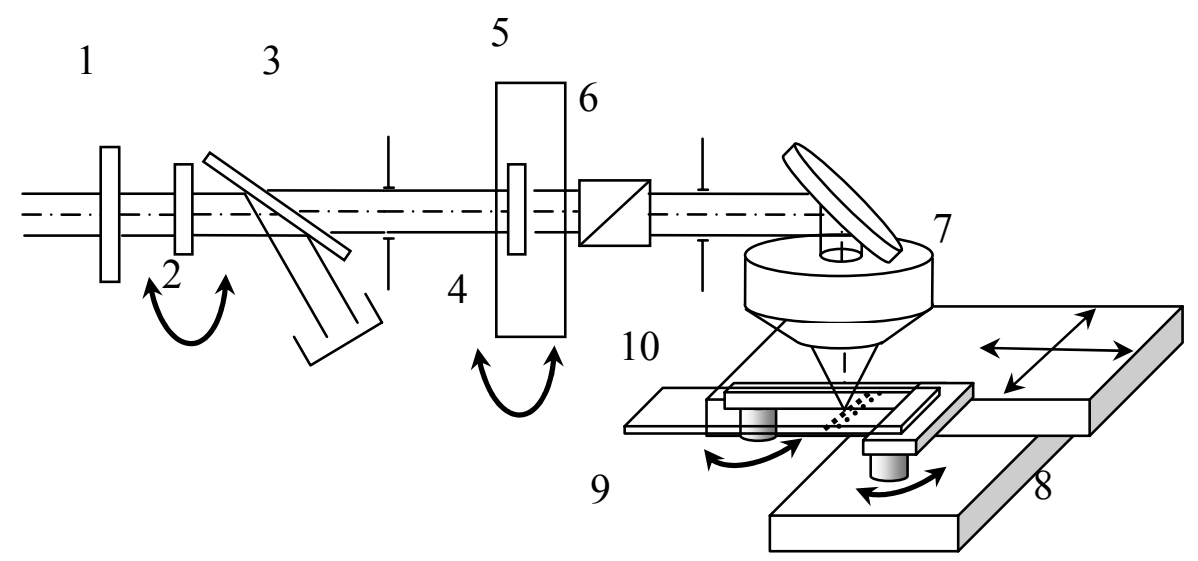

Fig. 1. Experimental set-up: after the shutter (1), half-waveplate (2), and Brewster angle thin-film polariser (3) a coarsely attenuated laser beam falls onto a second half-waveplate (4), mounted on a computer controlled rotation stage (5) (Physics Instruments). The femtosecond beam with the polarization defined by Glan prism (6) is focused by microscope objective (7) onto a front surface of a sample (10), coated with Al. The sample is mounted on a modified mirror mount (9) and 2D precision translation stage (8).

Several trial runs were carried out to determine the optimum laser energy, number of scans and writing speed for laser ablation of metal films with different thicknesses. We found that it was difficult to generate a good quality ablation track while leaving the glass surface untouched at any laser pulse energy when a thick coating (about $1000 \mathrm{~nm}$ ) was used. This is the task where the advantage of the multilayer coating approach [8-10] becomes obvious. In our experiments, while higher energies produced deeper ablation spots, to avoid damage, the speed had to be increased, while with lower energies there was insufficient penetration of the beam for etching, even at much lower speed. Furthermore, when the speed was sufficiently high ( $>2 \mathrm{~mm} / \mathrm{s})$, it resulted in a discontinuous line as the ablated areas of the Al coating did not adequately overlap. Other problems affecting direct laser ablation are variations in thickness of the metal film and the deviation in pulse to pulse energy of the laser. These may result in non-ablated parts of the track, thus affecting ion exchange in those areas, or variable width of the ablated strip, leading to parasitic scattering and reflection for the guided light, and thus to a high propagation losses.

To overcome these variability issues we implemented a multiple scanning technique, which was proven to produce uniform and reliable ablation patterns (Fig. 2). A typical ablation stage includes a first scan with laser pulse energy in the range of 50-150 nJ, followed by a one to three successive scans with gradually decreasing energy - down to 20-30 nJ. These are typical parameters for a $100 \mathrm{~nm}$ thick Al coating and constant scanning speed of $0.1-0.2 \mathrm{~mm} / \mathrm{sec}$. The proper choice of the energy level at a suitable velocity produced consistently reproducible line widths for a given aluminium thickness (Fig. 2d).

Periodic structures can also be fabricated (Fig. 3) with this technique either by creating groves in the glass surface or by introducing a periodic structure in the metal film and thus index variation after ion-exchange. The former seem to offer better sensitivity to parameters in the adjacent medium, while the later could produce a smooth and "almost sinusoidal" index variation along the track due to $\mathrm{Ag}$ indiffusion, embedded below the surface, but, with a lower coupling coefficient. In single shot laser ablation experiments [14] it was demonstrated that clear holes as small as $\sim 40 \mathrm{~nm}$ can be fabricated. The limiting factors here are the spot size (the minimum is about laser wavelength) and the above thickness of the metal layer used. While our focal spot can be successfully tailored down to sub-micrometer region, the metal layer has to be sufficiently thick to offer its protective function. So far, using a x36 objective we been able to produce holes on 
a metal layer with a minimal period of about 3 um and in single shot experiments we have been able to ablate holes, estimated from relative transmission measurements, to be as small as $\sim 100 \mathrm{~nm}$. We did try another approach $[15,16,18]$, well fitted for a short-period pattern formation. There is strong evidence (Fig. $3 \mathrm{~b}, \mathrm{c}$ ) that the period can be tailored down to few hundred nanometers [15 - 18]. Alternative approaches were also demonstrated in $[19,20]$.
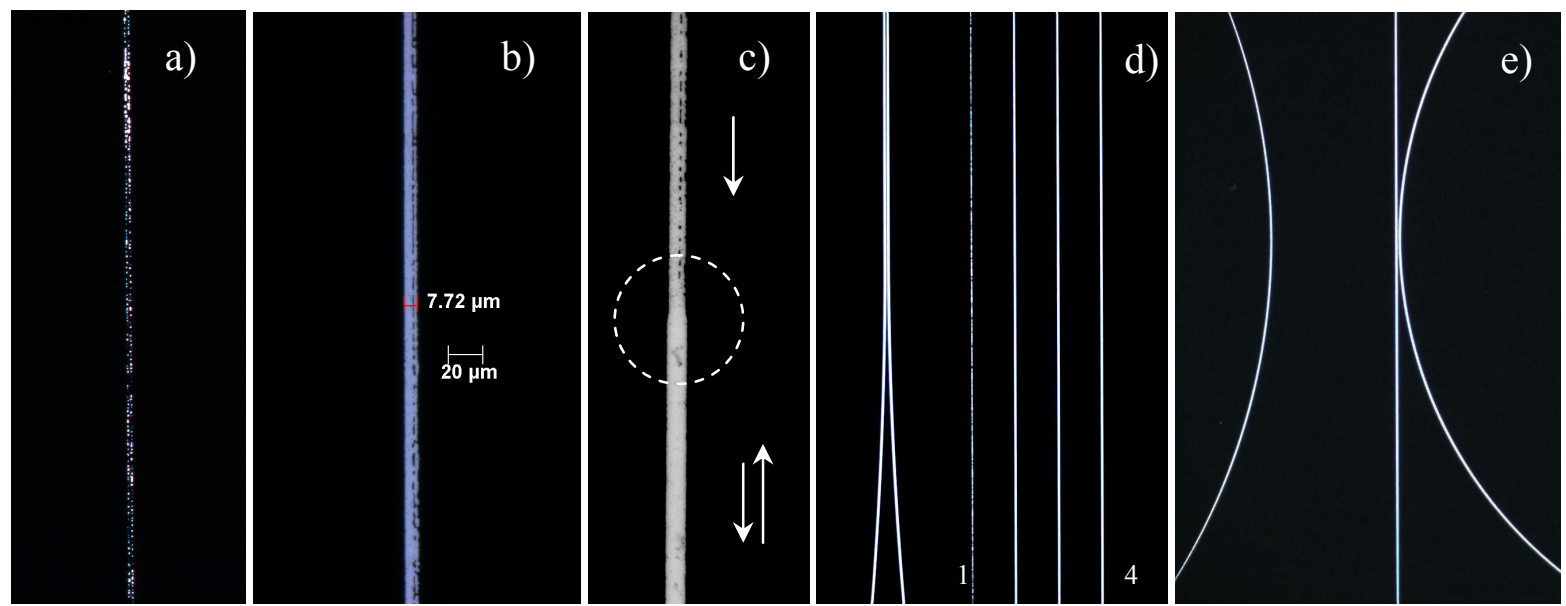

Fig. 2. The results of the ablation stage of the aluminium film, when: a) laser pulse energy is too small; b) laser pulse energy is too large, the re-deposition of the aluminium onto ablated strip is seen; c) second scan of the laser removes redeposited metal and leaves clean trace behind; d) the lines from 1 to 4 represent the influence of the number of scans with the same energy - from 1 to 4 respectively. Y-coupler from the left was made with two scans; e) the result of ablation of ring-resonator structure with the optimised parameters.
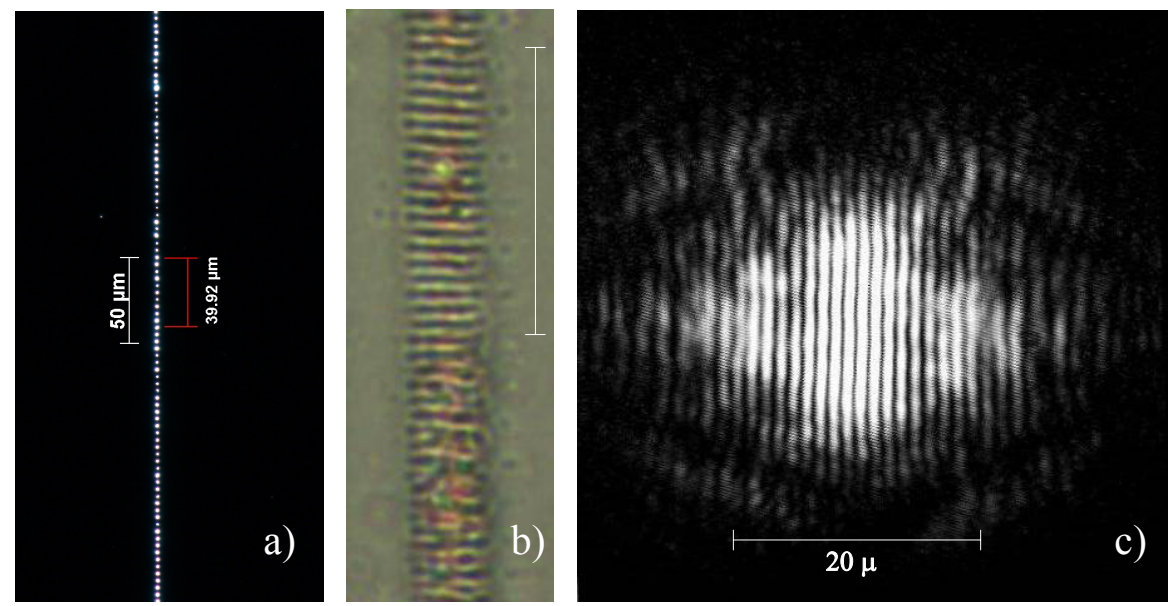

Fig. 3. Periodic perforation of a line of holes with 4 micrometers period on aluminium film (a) revealed poor uniformity. Photo of the glass with a surface structure of $1 \mu$ period (b), fabricated using a micro-holographic method [15 - 18]. Intensity distribution in the plane, with the maximum fringe contrast (c) [17]. A scale bars on both (b) and (c) correspond to $20 \mu \mathrm{m}$.

In our experiments, thermal ion-exchange was carried out in a dilute melt of $\mathrm{NaNO}_{3}$ and $\mathrm{AgNO}_{3}$. The concentration

of silver ions was $\sim 10^{-3}$. This allowed for better control over diffusion rates, and thus index contrast, for producing single mode waveguides, as against using a pure melt of $\mathrm{AgNO}_{3}$. The diffusion process makes smoother short scale structures, however we found it is sensitive to the sample uniformity, both natural and induced. The temperature of the exchange was carried out in a furnace at $360^{\circ} \mathrm{C}$ typically for 45 minutes. The adhesion of the metal film and its ability to 
withstand the elevated temperatures are of great importance. As ion-exchange is a diffusion process, one may expect that minor defects of the ablation are significantly washing out. At the same time, tiny and invisible micro-pores on a metal film become much clearer, especially in the area adjacent to the track, as the film here experienced more thermal stresses (Fig. 4). We believe that the reason for this is bad adhesion of the $\mathrm{Al}$ film in our samples. After the ion-exchange stage those spots adjacent to the waveguide became much more pronounced. On Figure 4, b the DIC examining of the resulting waveguide (after the ion-exchange and polishing) revealed the following faults of the process: i) overexposure of the glass surface - as the track has parallel lines on it because of different diffusion in pristine and illuminated glass; ii) the "bumps" adjacent to waveguide, perhaps, due to bad adhesion, will cause high propagation losses; iii) cracked edge of the sample may cause high coupling losses; iv) other defects on the metal coating, where no laser ablation took place, probably due to poor cleaning. These defects are a major contribution to the observed high propagation losses in the waveguide.
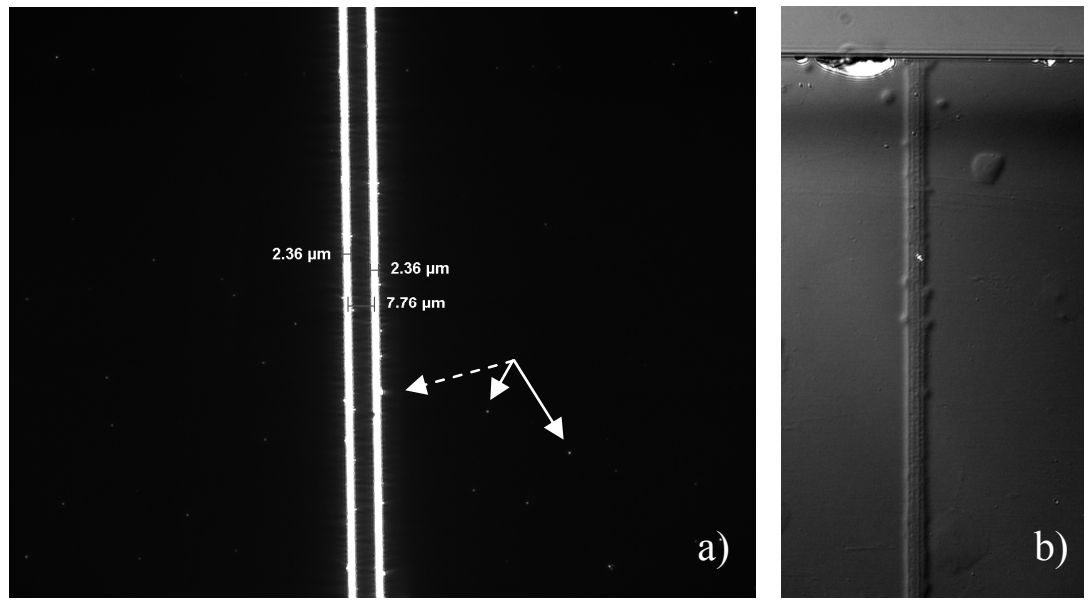

Fig. 4. Bad adhesion of the $\mathrm{Al}$ film manifests itself as sporadically appeared dots in places where no laser ablation was done (marked on (a) with white arrows). The track, even if it looks after ablation "clean", may have some spots adjacent to it (marked by dashed arrow) appeared after the ion-exchange stage. This is most pronounced if ultrasonic bath cleaning was used.

Though in our work we have used soda-lime glass [13], it is possible to use other hosts, such as lithium niobate and other speciality glasses, including active ion-doped materials.

\section{RESULTS AND CHARACTERISATION}

Prior to ion-exchange, the patterned structures were examined under an optical microscope to estimate their linewidths as well as to check for any defects. Linewidths of the ablated track measured on some of the patterned structures is shown in Figs. 2. Typically, it is below $6 \mu \mathrm{m}$, and the waveguide width becomes between 6 to $10 \mu \mathrm{m}$ depending on the duration of the ion-exchange stage.

After ion-exchange, the aluminium mask was removed and the sample was again examined under the microscope (Fig. 5). A Differential Interference Contrast microscope was used to identify the regions of higher refractive index obtained after the ion-exchange stage from the rest of the glass. Refractive index contrast of the waveguides was measured using the method of Quantitative Phase Microscopy (QPM), the results are presented on Fig. 5 (and insert on Fig 5, b). Typically, for devices of interest the optimal values were in the region of 0.005 to 0.008 . With some modifications of the ion-exchanged parameters, it is possible to produce at least one order of magnitude higher index contrast than that has been possible in this work. The exact value can be verified from reconstruction of the refractive index profile from nearfield intensity distribution measurements which is presently work in progress. 

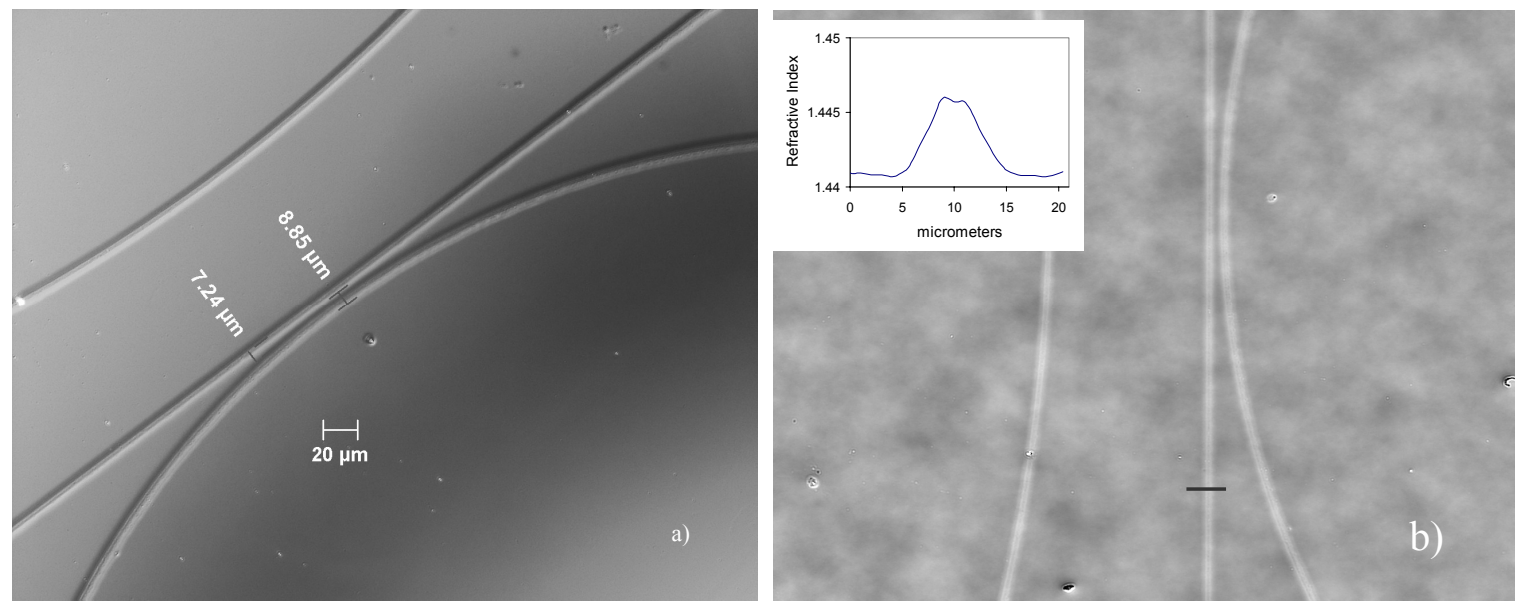

Fig. 5. a). Sample showing straight and curved waveguides obtained by the process described. The aluminium mask has been removed after ion-exchange. DIC (Nomarsky) image of the ring-resonator structure; b). Map of refractive index (phase retardation) obtained using the QPM method (on insert - the refractive index contrast measurements across the waveguide formed as shown by the black line).

The V-parameter of the straight waveguide was estimated (assuming step-index profile with the contrast of 0.005 and radius of the waveguide of $3.5 \mu \mathrm{m}$ ) to be $\sim 3.7$ at He-Ne laser wavelength. While the waveguides should be multimode at both 532 and $633 \mathrm{~nm}$, there is a strong indication of single mode propagation at $1550 \mathrm{~nm}(\mathrm{~V} \sim 1.8)$, which is the region of interest for most device operations in communication and sensing.
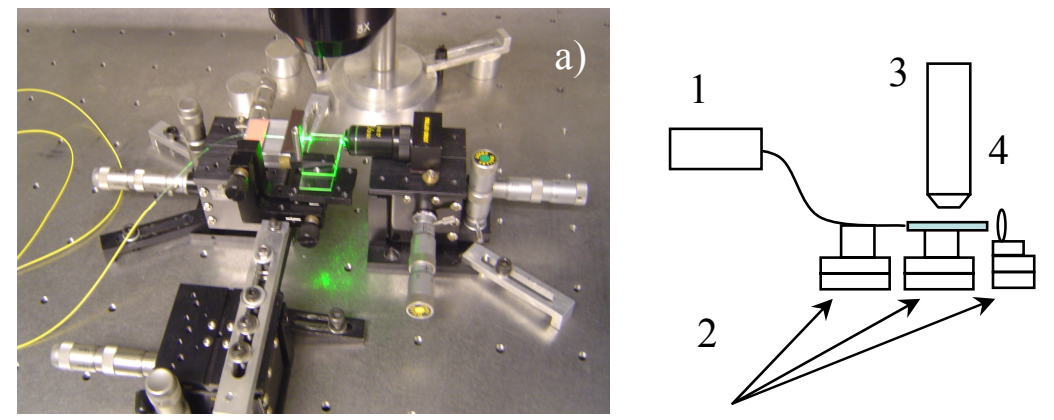

b)
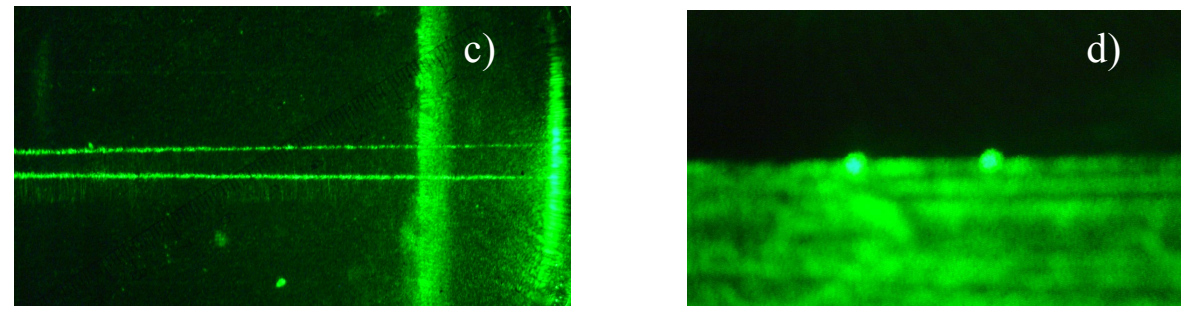

Fig. 6. The coupling set-up (a, b) consists of laser source (1) coupled to the optical fibre, three micro-positioning stages (2) to manipulate in-, output fibre ports, as well as a sample. The coupling was accomplished under a stereo microscope (3). A top view on Y-coupler is presented on Fig. c. The output fibre port can be changed to a microobjective (4), to image the output surface of a device (like Y-coupler on Fig. c) onto a screen (5), or a camera (Fig. d) for further analysis. 
In order to couple light into the waveguide, the edges of the sample were polished. First the sample was cut close to the edges by a precision saw (Model 15, Logitech). The sample was then bonded along with dummy slides and polished until the surface produced no defects on the waveguide edges as was observed under the optical microscope.

The set-up used for butt-coupling of the waveguide is shown in Fig. 6. It consisted of a laser source with a fibre pigtail that butt-couples light into the input edge of the waveguide. At the output edge, a x 25 microscope objective was mounted to couple light out and create an image of the output plane on the CCD. The near-field pattern of the edge was captured on the screen at the output end. The fibre pigtail, sample and microscope objective were all mounted on precision XYZ translation stages and were suitably manipulated for maximum coupling. The middle stage has a modified mirror mount at the end of the extended holder, which was used to make fibre and waveguide to be collinear. Furthermore, a microscope was placed above the sample to visually observe the path of the waveguide due to light scattering. This arrangement was also useful in identifying the waveguide input and the output edges that enhanced coupling capability. The scattered images of the edge coupling on some of the waveguides are shown in the Fig. 6, where the image of guided light at the output ports of Y-coupler is also presented.

The transmission losses were measured by side scattering technique and found to be below $6 \mathrm{~dB} / \mathrm{cm}$ at the wavelength of $532 \mathrm{~nm}$ for poor quality waveguide shown on Fig. 4, b, of approximately $20 \mu \mathrm{m}$ in width. Coupling losses were estimated to be up to $6 \mathrm{~dB}$ per surface, because of the mismatch of the modes diameter between the SMF-28 fibre and test waveguide (these have $20 \times 5 \mu \mathrm{m}$ cross section in our first experiments). The propagation losses for the waveguide on Fig. 5, a seem to be less than for waveguide shown on Fig. 4, b, as the side scattering observed in microscope was lower.

We believe that the main sources of propagation losses are stochastic variation of the waveguide cross-section and the surface corrugation left after laser ablation stage. We are planning to implement two stage ion-exchange process with the field assisted $\mathrm{Ag}$ indiffusion (in another salt melt) being the second stage with the aim of achieving state of the art performance for the ion-exchange waveguides: propagation losses of a single-mode one - less than $0.01 \mathrm{~dB} / \mathrm{cm}$, circular mode-field distribution, perfectly matching the mode of a standard fibre and positioned at the depth of up to 20 micrometers.

\section{CONCLUSION}

We have successfully fabricated optical waveguides by combining a direct femtosecond laser ablation with ion-exchange method. The laser energy level and speed of writing has been optimised for our system to produce consistently reproducible patterns that can be used for fabrication of ion-exchange waveguides in glass. Optimal index contrasts in the region of 0.005 to 0.008 have been obtained by this combination technique which allows the flexibility and ease of prototyping of photonic devices as compared to exclusively using direct laser writing method or by conventional lithography. Low-loss and high contrast waveguides can be achieved by this method and would have applications in sensing and telecommunication.

The authors acknowledge the financial support of the Engineering \& Physical Sciences Research Council in carrying out this work.

\section{REFERENCES}

[1] Nishihara, H., Haruna, M., Suchara, T., [Optical Integrated Circuits], McGraw-Hill Professional (1989).

[2] Hunsperger, R.G., [Integrated Optics], Springer-Verlag (2002).

[3] Murphy, E.J., [Integrated Optical Circuits and Components: Design and Applications], Marcel Dekker (1999).

[4] Najafi, S. I., [Introduction to Glass Integrated Optics], Artech House, Boston (1992).

[5] Eldada, L., "Polymer integrated optics: Promise vs. practicality," Proc. SPIE 4642, 11-22(2002).

[6] Brinker, C.J., and Scherer, G.W., [Sol-Gel Science: The Physics and Chemistry of Sol-Gel Processing], Academic Press (1990).

[7] Corin, B. E., Fu, G.A., Bhutta, T., Hua, P., Shepherd, D.P., Taylor, E.R., and Smith, P.G.R., "Direct-UV-written buried channel waveguide lasers in direct-bonded intersubstrate ion-exchanged neodymium-doped germanoborosilicate glass," Appl. Phys. Lett. 81(19), 3522-3524 (2002). 
Salguerio, J.R., Roman, J.F., and Moreno, V., "System for laser writing to lithograph masks for integrated optics," Optical Engineering 37(4), 1115-1123 (1998).

[9] Koch, J., Fadeeva, E., Engelbrecht, M., Ruffert, C., Gatzen, H.H., Ostendorf, A., and Chichkov, B.N., "Maskless nonlinear lithography with femtosecond laser pulses," Appl. Phys. A. 82, 23-26 (2006).

[10] Chichkov, B.N., Fadeeva, E., Koch, J., Ostendorf, A., Ovsianikov, A., Passinger, S., Reinhardt, C., "Femtosecond laser lithography and applications," Proc. SPIE 6106, 610612 (2006).

[11] Grobnic, D., Mihailov, S.J., Smelser, C.W., Walker, R., "Bragg gratings made with ultrafast radiation in non-silica glasses; fluoride, phosphate, borosilicate and chalcogenide Bragg gratings," Proc. SPIE 6796, 67961K (2007).

[12] Vorobyev, A.Y., and Guo, C., "Change in absorptance of metals following multi-pulse femtosecond laser ablation," Journal of Physics: Conference Series 59, 579-584 (2007).

[13] Menzel Glaser, http://www.menzel.de.

[14] Joglekar, A.P., Liu, H., Meyhöfer, E., Mourou, G., and Hunt A.J., "Optics at critical intensity: Applications to nanomorphing, " PNAS 101(16), 5856-5861 (2004).

[15] Maznev, A. A., Crimmins, T. F., and Nelson, K. A., "How to make femtosecond pulses overlap," Opt. Lett. 23(17), 1378-1380 (1998).

[16] Kondo, T., Matsuo, S., Juodkazis, S. and Misawa, H., "Femtosecond laser interference technique with diffractive beam splitter for fabrication of three-dimensional photonic crystals," Appl. Phys. Lett. 79(6), 725-727 (2001).

[17] Dubov, M., Nasyrov, R. K., Nikogosyan, D. N., Poleshchuk, A. G., Mezentsev, V., and Bennoin, I.,"Submicrometer grating fabrication in fused silica with UV femtosecond laser," to be published (2008).

[18] Paivasaari, K., Kaakkunen, J. J. J., Kuittinen, M., and Jaaskelain, T., "Enhanced optical absorptance of metals using interferometric femtosecond ablation," Optics Express 15(21), 13838-13843 (2007).

[19] Pissadakis, S., Pappas, C., "Planar periodic structures fabricated in Er/Ybcodoped phosphate glass using multi-beam ultraviolet laser holography," Optics Express 15(7), 4296-4303 (2007).

[20] Dubov, M., Mezentsev, V., Bennion, I., and Nikogosyan, D.N., "UV femtosecond laser inscribes a $300 \mathrm{~nm}$ period nanostructure in a pure fused silica," Meas. Sci. Technol. 18(7), L15-L17 (2007). 